\title{
COPING STRATEGIES IN THYROID DISEASES
}

\author{
ANNAMÁRIA PÁPAI ${ }^{1}$, MARIA MELANIA COZMA ${ }^{2}$, LUCICA EMILIA COȘA ${ }^{3}$, \\ ADRIANA MIHAI ${ }^{4}$ \\ 1234 “George Emil Palade” University of Medicine, Pharmacy, Science, and Technology of Târgu Mureş
}

Keywords: stress, thyroid diseases, mechanism, psychotherapy

Abstract: In the present study, we aimed at evaluating the relationship between the cognitive coping strategies of people with thyroid diseases and the level of stress experienced by them. During the present study we evaluated the coping strategies with Cognitive Emotion Regulation Questionnaire, and the level of stress experienced by them with Holmes and Rahe stress scale. All patients attended an endocrinology outpatient clinic between may-august 2019. 42 thyroid patients (31 with hypothyroidism and 11 with hyperthyroidism), aged 33-69 were selected for this study. The coping strategies used predominantly by thyroid patients are: rumination, positive reappraisal, catastrophizing. The rumination and the level of stress experienced correlated positively $.295 *, p=$ .044 , statistically significant $(p<0.05) .58 .13 \%$ of patients presented stressful events in the year prior onset of thyroid pathology. This finding is important because restructuring less proactive coping strategies through psychotherapies can be an effective alternative or adjuvant way of treating thyroid diseases.

\section{INTRODUCTION}

The research studies $(1,2,3)$ have highlighted an important role of stressors in determining, occurring and maintaining thyroid diseases, but there is little information regarding the manner and the mechanism how these events and stressors mediate thyroid diseases. Patients with Graves' disease often report a stressful event before the onset of the disease. $(4,5,6,7)$ This is usually a serious negative event, such as an accident, loss of a relative or of a job. Stress also plays an important role in autoimmune thyroiditis.(1)

Our brain is programmed to perceive and catalog the experiences lived as negative or dangerous, neutral or positive, and the reaction is in concordance with this label. Fight reaction are reflected by the facial expression of the anger, the verbal or physical aggression; while the social isolation, the development of some addictions (computer, games, substances etc.) are specific to avoidance reactive behaviors. Freeze reaction, that of stumbling, the inability to act, characterized by the lack of physical or mental reactions such as the feeling of helplessness, depressive symptoms is the third possibility. If the situation is not resolved by freeze, fight or fly (avoidance), and the individual remains exposed to stressful agents; over time, emotional disturbances, restlessness, persistent high physical and mental stresses appear that eventually weaken the immune system and cause cardiovascular, endocrine and psychical diseases, cancer, diabetes etc.

If the role of stress has been mentioned in specialized studies, the role of coping strategies in thyroid diseases has not been studied yet, although in addressing stress as a trigger factor for thyroid diseases coping strategies (the ways to react to stress) play an important role.

But what is coping actually? A definition of coping is provided by Monat and Lazarus (8) who define coping as "a person's efforts to meet the requirements (conditions of injury, threat or different challenges) that are evaluated (or perceived) as exceeding or overloading their own resources." The specialty literature outlines two functions of coping: problem-centered coping that is directly oriented to the stressor- and emotionfocused coping, which refers to the regulation of emotions that appear as a result of the stressor.(9) Coping strategies that focus on problem solving are considered more effective than emotionfocused coping strategies.(10)

The regulation of emotions through cognates is a basis of cognitive behavioral and emotional therapy. Cognitive processes help people regulate their emotions in a way that they are not overwhelmed by the intensity of emotions and feelings experienced during negative events. Cognitive processes can be divided into unconscious cognitive processes: projection or denial, and conscious cognitive processes such as: self-blame, blaming others, rumination and catastrophizing.(11)

In our present study we focused on the cognitive aspects of coping such as: self-blame, acceptance, rumination, positive refocusing, refocusing on planning, positive reappraisal, putting in perspective, catastrophizing, blaming others. By establishing the strategies of cognitive coping of people with thyroid diseases, we will be able to design the psychological intervention programs of cognitive behavioral and emotional type, more efficiently.

The study is an important step in the construction of the psychotherapeutic intervention program, through which we want to restructure both behavioural coping and cognitive coping strategies in people with thyroid diseases, offering an alternative psychotherapeutic treatment in the treatment of thyroid diseases, especially of the psycho-emotional components

${ }^{1}$ Corresponding author: Annamária Pápai, Str. Lalelelor, Nr. 11, Târgu-Mureş, România, E-mail: annamariaporkolab@yahoo.com, Phone: +40745 315332

Article received on 08.04 .2020 and accepted for publication on 28.05.2020 
associated with them.

\section{AIM}

As an objective of the present study, we aimed at evaluating the cognitive coping strategies of people with thyroid diseases (hypo- and hyperthyroidism) and the level of stress experienced by them, at the same time we followed if there are relationships between the level of stress experienced and the coping strategies used by them.

\section{MATERIALS AND METHODS}

In our retrospective study we included 42 patients with thyroid disease (31 with hypothyroidism and 11 with hyperthyroidism), aged 33-69. All patients attended an endocrinology outpatient clinic between may-august 2019, who accept to participate at the study and fulfil selection criteria were admitted. Written consent was obtained from all participants.

Inclusion criteria were the following: patients diagnosed with thyroid disease according with a specialist endocrinologist in last three years and/ or TSH, FT4 with altered values. Exclusion criteria were the following: patients without a specialist diagnosis, more than 3 years from onset of disease, incapacity to write or to understand the test demands. The questionnaire (Cognitive Emotion Regulation Questionnaire and Holmes and Rahe stress scale) was applied - after the diagnosis, before treatment and during treatment.

For the classification of people in the category of thyroid patients we used blood tests that revealed the levels fT3, fT4, TSH, as well as the diagnosis established by the attending physician.

During present study we evaluated the cognitive and emotional coping strategies of thyroid patients (Cognitive Emotion Regulation Questionnaire), the level of stress experienced by them (Holmes and Rahe stress scale), at the same time following the relationships between the level of stress experienced and the coping strategies used (self-blame, acceptance, rumination, positive refocusing, refocus on planning, positive reappraisal, putting into perspective, catastrophizing, blaming others).

In this study it was not important how severe hyperthyroidism was or if the patient is after or in a medical treatment because the coping strategies, the way how we react to stressful events, do not change due to endocrinological medication.

In the present study we applied the following tests: Cognitive Emotion Regulation Questionnaire (CERQ), Holmes and Rahe stress scale. Participants gave pen- a- paper responses. The Cognitive Emotion Regulation Questionnaire (CERQ) is a very effective diagnostic tool regarding the possibility of highlighting the extent to which a person uses adaptive or maladaptive cognitive coping strategies when facing negative events.(12) Compared with other questionnaires of coping that do not make a clear distinction between a person's thoughts and work, CERQ refers exclusively to the thoughts that you have experienced as a negative experience. CERQ questionnaire measuring frequency of use of certain strategies through 9 rating scales: self-blame, acceptance, rumination, positive refocusing, refocus on planning, positive reappraisal, putting into perspective, catastrophizing, blaming others.

With the Holmes and Rahe scale we evaluated the stressful events of the past five years of patients. Holmes and Rahe (11) demonstrates how stressful life events affect physical and emotional health of those exposed. The two authors built on a scale observation with 43 events each event having a particular score depending on the influence it had on people. To measure the level of stress in a person's life, you have to gather point values for each change and the final score will be the level of stress.

The data from the questionnaires were processed quantitatively with the SPSS 22 statistical program: descriptive analyzes: frequency, means, correlations, Anova analysis, Shapiro-Wilk test, Post-Hoc, Bonferoni and Tamhane tests. We also used the comparison of the averages of coping strategies of the people with thyroid diseases with the average level calibrated on the Romanian population.(12)

\section{RESULTS}

The coping strategies used predominantly by people with thyroid disease compared to the average of Romanian population (12) are: rumination and catastrophizing. People with thyroid diseases also use proactive or functional coping strategies such as accepting the situation, which is used more often by people with hyperthyroidism. People with hypothyroidism are characterized by coping strategies such as: self-blame, catastrophizing, refocusing on planning and accepting the situation. The coping strategies characteristic to people with hyperthyroidism are: rumination, catastrophizing, accepting the situation and positive reappraisal.

Table no. 1. Results of Cognitive Emotion Regulation Questionnaire (CERQ)

\begin{tabular}{|l|l|l|l|}
\hline \multicolumn{1}{|c|}{ Coping strategies } & $\begin{array}{c}\text { Mean } \\
\text { hyperthyroid }\end{array}$ & $\begin{array}{c}\text { Mean } \\
\text { hyperthyroid }\end{array}$ & $\begin{array}{c}\text { Average } \\
\text { Ro }\end{array}$ \\
\hline Self-blame & 11,29 & 9,67 & 8,22 \\
\hline Acceptance & 12,29 & 13,06 & 11,03 \\
\hline Rumination & 12,43 & 12,56 & 10,50 \\
\hline Positive refocusing & 10,29 & 11,33 & 9,91 \\
\hline $\begin{array}{l}\text { Refocusing on } \\
\text { planning }\end{array}$ & 15,57 & 13,78 & 13,08 \\
\hline Positive reappraisal & 14,14 & 14,56 & 12,45 \\
\hline $\begin{array}{l}\text { Putting into } \\
\text { perspective }\end{array}$ & $12,4,633$ & 13,44 & 11,63 \\
\hline Catastrophizing & 9,14 & 8,89 & 6,07 \\
\hline Blaming others & 8,29 & 7,56 & 6,37 \\
\hline
\end{tabular}

Regarding the stressful events experienced by people with thyroid diseases, it is noted that $58.13 \%$ of the sample concerned experienced events with a level of stress that, according to the applied scale, increase the risks of psychosomatic illness. The applied stress scale highlights four categories regarding the level of stress experienced, represented in table no.2.

Table no. 2. The level of stress experienced by people with thyroid disease

\begin{tabular}{|c|c|c|c|}
\hline $\begin{array}{c}\text { No major } \\
\text { problems }\end{array}$ & $\begin{array}{c}\text { Mild } \\
\text { stress }\end{array}$ & $\begin{array}{c}\text { Stress with chances of } \\
\text { developing illnesses }\end{array}$ & $\begin{array}{c}\text { Major } \\
\text { crisis }\end{array}$ \\
\hline $23.25 \%$ & $18.60 \%$ & $46.51 \%$ & $11.62 \%$ \\
\hline \multicolumn{2}{r}{ The statistical analysis revealed that there is a }
\end{tabular}
statistically significant correlation between rumination as a coping strategy and the stress experienced by people with thyroid disease $(.295 *, \mathrm{p}=.044)$. In order to respect the normality condition of the distributions at the level of the comparable groups, we checked them with the Shapiro-Wilk test which shows us a p (sig.) greater than 0.05 , in almost all of the four groups (except being the category mild stress, $\mathrm{p}=0.009$ ), which supports the conclusion of normality for the distributions at group level.

In people with mild stress, acceptance is higher, and the putting into perspective and positive reappraisal is lower than the average of people with thyroid diseases. People with stress with chances of developing illnesses have a high level for the coping strategy refocusing on planning. Rumination, catastrophizing and positive refocusing are high in people with a very high level of stress (major crisis). 
CLINICAL ASPECTS

Table no. 3. The average of coping strategies for people with thyroid diseases, according to the level of stress experienced

\begin{tabular}{|l|c|c|c|c|c|c|c|c|c|}
\hline \multicolumn{1}{|c|}{ Stress level } & SelfB & Acc & Rum & PRef & RefPlan & PosR & Pers & Cat & Bo \\
\hline $11-150$ & 10,58 & 13,42 & 9,33 & 11,00 & 14,67 & 16,08 & 14,42 & 8,17 & 7,67 \\
\hline $151-199$ & 10,44 & 14,11 & 12,11 & 11,67 & 14,56 & 14,22 & 11,67 & 7,78 & 7,44 \\
\hline $200-299$ & 10 & 13,14 & 12,95 & 11,71 & 15,38 & 14,76 & 13,43 & 7,67 & 7,29 \\
\hline over 300 & 10,8 & 13 & 14,4 & 16 & 13,8 & 15,4 & 14,6 & 8,6 & 7,6 \\
\hline AverageRo & 10,32 & 13,38 & 12,02 & 11,98 & 14,87 & 15,06 & 13,47 & 7,91 & 7,45 \\
\hline
\end{tabular}

(Key Stress level 11-150 -No major problems, 151-199 - Mild stress, 200-299 - Stress with chances of developing illnesses, over 300 - Major crisis)

(Key: SelfB - self-blame, Acc - acceptance, Rum - rumination, PRef - positive refocusing, RefPlan - refocusing on planning, PosR - positive reappraisal, Pers - putting into perspective, Cat - Catastrophizing, Bo - blaming others)

The result of the $\mathrm{F}$ test following the ANOVA analysis whose value is 2.886 for a significance threshold $\mathrm{p}=0.046$ indicates that the positive reappraisal as a coping strategy varies significantly, according to the categories of the Stress scale. Post-Hoc, Bonferoni and Tamhane test results, from multiple comparisons, show that the level of positive reappraisal of the subjects in the category no major problems differs significantly from the level of positive refocusing in the categories major crisis and stress with chances of developing illnesses $(\mathrm{p}=$ $0.041)$.

The analysis of homogeneity in the four groups of measured stress (no major problems, middle stress, stress with chances of developing illnesses, major crisis) was carried out by Levene's test. Its value is not significant $(p=0.060)$, we can conclude/ state that dispersions within the four target stress groups are homogeneous.

The Shapiro-Wilk test has a p less than 0.05 for each of the four groups, which supports the conclusion of normal distributions of stress levels measured in the groups.

The result of $F$ test (one-way ANOVA) whose threshold value is $p=4.067$ for a 0.013 allows rejection of the null hypothesis and accepting the hypothesis that rumination as a coping strategy varies depending on the level of stress experienced by the evaluated persons.

Following the post-hoc analysis (multiple comparisons) we find that the level of rumination as a coping strategy of subjects from no major problems category differs significantly from the level of rumination as a coping strategy categories of stress with chances of developing illnesses or major crisis. $(\mathrm{p}=0.025$, respectively 0.037$)$.

\section{DISCUSSIONS}

Regarding the cognitive, emotional coping strategies, which refer to the reactions and thoughts of the person after experiencing negative events, the following profiles of people with hyperthyroidism are outlined: rumination, catastrophizing, acceptance of the situation and positive reappraisal; people with hypothyroidism are characterized by coping strategies such as: self-blame, catastrophizing, refocusing on planning and accepting the situation. In general, people with thyroid diseases have very high scores on all nine coping strategies - self-blame, acceptance, rumination, positive reappraisal, refocusing on planning, positive refocusing, putting into perspective, catastrophizing, blaming others. Even though it has been shown from the statistical results that the most characteristic for people with thyroid diseases are rumination and catastrophizing, high scores above the population average, it was also highlighted in adaptive and functional coping strategies. These functional coping strategies, when they present high scores, can no longer be categorized as adaptive and functional, and they will signal some emotional problems.

According to the CERQ manual high scores of refocus on planning without taking action may be associated with certain emotional problems.(12)

Thus, high scores of rumination, with coping strategies such as refocusing on planning can increase psychosomatic symptomatology, because the person moves in a vicious circle that does not lead to the processing of negative events experienced; on the contrary, these can aggravate the emotional problems and even block the person and favour the occurrence of mental illnesses.

This information may be effective for people with thyroid diseases for establishing an alternative psychotherapeutic treatment. A psychotherapeutic treatment means eliminating maladaptive coping strategies and learning adaptive strategies.

In order to be able to help people with thyroid disease in restructuring cognitive coping strategies for coping effectively with negative events, we need to establish a model for combining these strategies according to the level of stress experienced by them.

Following the statistical analysis we can conclude that the majority of people with thyroid diseases, $58.13 \%$, have experienced stressful events that predispose to psychosomatic illness. The rumination coping strategy correlates positively with the level of stress experienced by people with thyroid diseases. The presence of symptoms of depression, anxiety or even suicide may indicate the use of maladaptive cognitive coping strategies, such as rumination, catastrophizing and selfblame.(13,14,15,16) In our study the first two of these maladaptive coping strategies were highlighted.

Our study shows the greater is the stress experienced in people with thyroid diseases, the more often positive refocusing strategy is used. From the results of the present research, it was also pointed out that the average score of the positive refocusing coping strategy in people with thyroid diseases is higher than of the average population in Romania, and, according to the test standard, (12) it represents a high score. The positive reappraisal coping strategy is a functional cognitive coping strategy, but this remains valid only if at the same time something is really done for solving the problem. Positive refocusing is a coping strategy whereby we think of pleasant things and not the event itself, which can also be a form of avoiding the problem itself. When people get a high score and do nothing to solve the problem, it will lead to emotional problems. And accepting as a coping strategy, with high scores, can also be a form of resignation, not just a functional strategy.

\section{CONCLUSIONS}

The experienced negative events increase the stress level of people, and, together with the less proactive coping strategies can be a part of the sources leading to thyroid diseases. Through the present study we managed to outline a profile of coping strategies for people with thyroid diseases, such as: people with hypothyroidism are characterized by coping strategies: self-blame, catastrophizing, refocusing on planning and accepting the situation, the coping strategies characteristic to people with hyperthyroidism are: rumination, catastrophizing, accepting the situation and positive reappraisal. Restructuring coping strategies through cognitive behavioural psychotherapies can be an effective alternative way of treating thyroid diseases. 


\section{REFERENCES}

1. Cojocaru M, Cojocaru IM, Silosi I. Multiple autoimunne syndrome. Maedica (Buchar). 2010;Apr:5.2:132-134.

2. Chrousos GP. Stress and disorders of the stress system. Nature reviews endocrinology. 2009;5.7:374.

3. Boscarino JA. Posttraumatic stress disorder and physical illness: results from clinical and epidemiologic studies. Annals of the New York Academy of Sciences. 2004;1032.1:141-153.

4. Sonino N, Girelli ME, Boscaro, et al. Life events in the pathogenesis of Graves' disease. A controlled study. Acta Endocrinol (Copenh). 1993;128:293-296.

5. Kung AW. Life events, daily stresses and coping in patients with Graves' disease. Clin Endocrinol (Oxf). 1995;42:303308.

6. Radosavljevi'c VR, Jankovic SM, Marinkovic JM Stressful life events in the pathogenesis of Graves' disease. Eur J Endocrinol. 1996;134:699-701.

7. Yoshiuchi K, Kumano H, Nomura S, et al. Stressful life events and smoking were associated with Graves' disease in women, but not in men. Psychosom Med.1998;60:182185.

8. Monat A, Lazarus RS, Stress and coping: An anthology (3rd ed.). New York. Columbia University Press; 1991. p. 5 .

9. Compas BE, Orosan PG, Grant KE. Adolescent stress and coping: Implications for psychopathology during adolescence. Journal of adolescence. 1993;16.3:331.

10. Thoits PA. Social psychology: The interplay between sociology and psychology. Social Forces. 1995;73.4:12311243.

11. Noone PA. The Holmes-Rahe Stress Inventory. Occupational Medicine. 2017;67(7):581-582.

12. Perte A, Tincas I. Handbook for use of the Cognitive Emotion Regulation Questionnaire - Adaptation and standardization of CERQ on the population in Romania. Cluj Napoca. ASCR Publishing; 2009. p. 468-502.

13. Garnefski N, Kraaij V, Spinhoven Ph. Negative life events, cognitive emotion regulation and depression. Personality and Individual Differences. 2001;30:1311-1327.

14. Garnefski N, Kraaij V, Spinhoven Ph. The relationship between cognitiv coping strategies and symptoms of depression, anxiety and suicidality. Gedrag en Gezondheid. 2001;29:166-176.

15. Garnefski N, van den Kommer T, et al. The relationship between cognitive emotion regulation strategies and emotional problems. European Journal of Personality. 2002; 16:403-420.

16. Garnefski N, Legerstee J, van den Kommer $\mathrm{T}$, et al. Cognitive coping strategies and symptoms of depression and anxiety: a comparison between adolescents and adults. Journal of Adolescence. 2003;25:603-611. 